\title{
Operator approach to effective medium theory to overcome a breakdown of Maxwell Garnett approximation
}

\author{
Popov, Vladislav; Lavrinenko, Andrei; Novitsky, Andrey
}

\section{Published in:}

Physical Review B

Link to article, DOI:

10.1103/PhysRevB.94.085428

Publication date:

2016

Document Version

Publisher's PDF, also known as Version of record

Link back to DTU Orbit

Citation (APA):

Popov, V., Lavrinenko, A., \& Novitsky, A. (2016). Operator approach to effective medium theory to overcome a breakdown of Maxwell Garnett approximation. Physical Review B, 94(8), [085428].

https://doi.org/10.1103/PhysRevB.94.085428

\section{General rights}

Copyright and moral rights for the publications made accessible in the public portal are retained by the authors and/or other copyright owners and it is a condition of accessing publications that users recognise and abide by the legal requirements associated with these rights.

- Users may download and print one copy of any publication from the public portal for the purpose of private study or research.

- You may not further distribute the material or use it for any profit-making activity or commercial gain

- You may freely distribute the URL identifying the publication in the public portal 


\title{
Operator approach to effective medium theory to overcome a breakdown of Maxwell Garnett approximation
}

\author{
Vladislav Popov* \\ Department of Theoretical Physics and Astrophysics, Belarusian State University, Nezavisimosti avenue 4, 220030, Minsk, \\ Republic of Belarus \\ Andrei V. Lavrinenko ${ }^{\dagger}$ \\ DTU Fotonik, Technical University of Denmark, rsteds Plads 343, DK-2800 Kongens Lyngby, Denmark
}

\begin{abstract}
Andrey Novitsky
Department of Theoretical Physics and Astrophysics, Belarusian State University, Nezavisimosti avenue 4, 220030, Minsk, Republic of Belarus

and DTU Fotonik, Technical University of Denmark, rsteds Plads 343, DK-2800 Kongens Lyngby, Denmark
\end{abstract}

(Received 2 June 2016; revised manuscript received 8 August 2016; published 26 August 2016)

\begin{abstract}
We elaborate on an operator approach to effective medium theory for homogenization of the periodic multilayered structures composed of nonmagnetic isotropic materials, which is based on equating the spatial evolution operators for the original structure and its effective alternative. We show that the zeroth-, first-, and second-order approximations of the operator effective medium theory correspond to electric dipoles, chirality, and magnetic dipoles plus electric quadrupoles, respectively. We discover that the spatially dispersive bianisotropic effective medium obtained in the second-order approximation perfectly replaces a multilayered composite and does not suffer from the effective medium approximation breakdown that happened near the critical angle of total internal reflection found previously in the conventional effective medium theory. We establish the criterion of the validity of the conventional effective medium theory depending on the ratio of unit-cell length to the wavelength, the number of unit cells, and the angle of incidence. The operator approach to effective medium theory is applicable for periodic and nonperiodic layered systems, being a fruitful tool in the fields of metamaterials and subwavelength nanophotonics.
\end{abstract}

DOI: 10.1103/PhysRevB.94.085428

\section{INTRODUCTION}

Metamaterials is the effective approach in condensedmatter physics for designing novel composite materials with exceptional electromagnetic (acoustic) properties that do not occur naturally [1]. The first demonstration of the negative refractive index behavior predicted by Veselago [2] was done with split-ring resonators employed as meta-atoms [3]. Currently, there is an extended nomenclature of various metallic, graphene, and high-index dielectric inclusions applied for designing metamaterials in microwave, terahertz, infrared, and optical ranges [4-17].

Typically, to treat a periodic composite as a continuous medium, the working wavelength should be much greater than the lattice period. Then fields within the unit cell are local, and material properties are determined by the polarization and magnetization vectors, i.e., by the dielectric permittivity and magnetic permeability tensors. The procedure of substituting an inhomogeneous structure with its homogeneous analog, which performs effectively the same, is called homogenization. As sketched in Fig. 1(a), the homogenized medium can acquire bianisotropic properties, which can include, in general, chirality and spatial dispersion.

\footnotetext{
*physics.vlad@gmail.com

†alav@fotonik.dtu.dk

$\ddagger$ anov@fotonik.dtu.dk
}

There are different approaches to find material parameters of a metamaterial, which can be grouped into the retrieval and homogenization methods. A retrieval method allows finding material parameters using reflection and transmission data $[18,19]$. These data can be obtained experimentally and/or from numerical simulations. Although this technique has certain drawbacks and constraints, e.g., ambiguity of the retrieval, difficulties with gathering the phase information in experiments, etc., these problems can be, in principle, solved. For example, an alternative wave-propagation retrieval method $[20,21]$ does not suffer from the ambiguity and can be applied to thick metamaterials. Usually, the parameters are retrieved accurately away from resonances, but in the vicinity retrieval suffers from enhanced errors and noises. In Ref. [22] the generalized retrieval method functioning near resonances was proposed.

Plenty of homogenization methods have appeared over the last decade as a reaction to the absence of a universal procedure applicable to a vast number of cases [23]. To overcome the limitations of the standard homogenization the first-principles homogenization theory for periodic metamaterials, the effective material parameters of which are spatially dispersive, was proposed in Ref. [24]. The multipole approach developed in Refs. [25,26] allows us to describe analytically bulk and planar metamaterials. The microscopic approach based on the dynamics of the classical electrons was used in Ref. [27] to describe the asymmetric transmission in planar chiral metamaterials [17]. Reference [28] deals with 

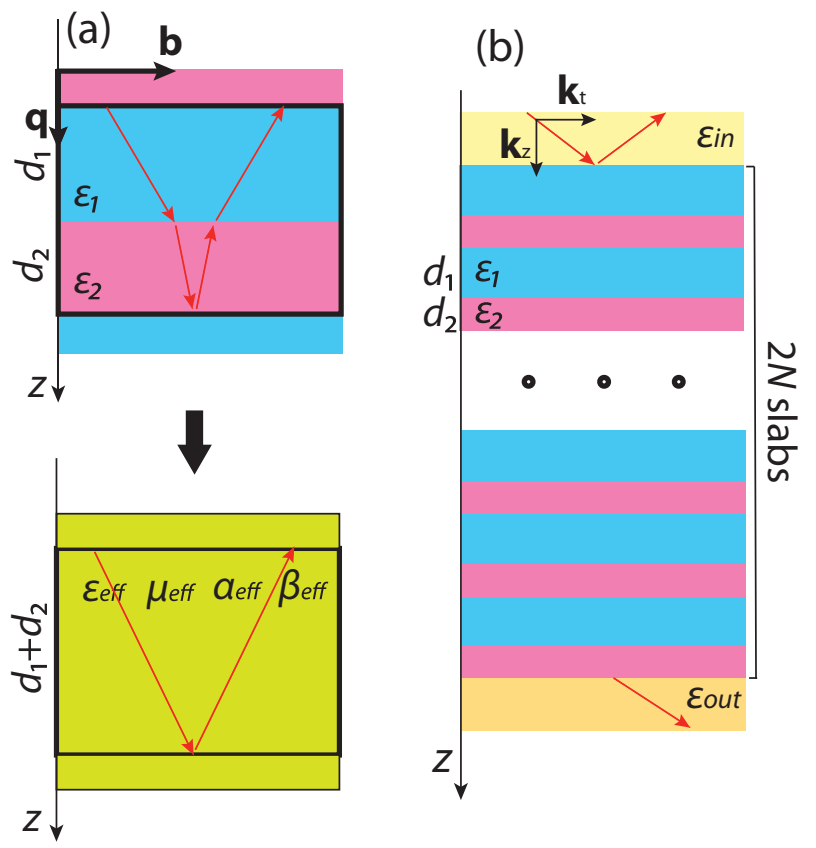

FIG. 1. (a) Bilayer unit cell of a multilayer dielectric structure (top) and its homogenized counterpart with bianisotropic effective medium parameters (bottom). (b) Periodic multilayered dielectric structure with $N$ bilayer unit cells.

electromagnetostatic homogenization of materials with microscopically bianisotropic inclusions. A rigorous homogenization theory via the Whitney interpolation developed in Ref. [29] allows us to reproduce the well-known Maxwell Garnett formula and introduce artificial magnetism.

Local homogenization can be insufficient for correct description of the wave propagation in a metamaterial even in its validity range. In this case, the spatial dispersion should be taken into consideration, and homogenization becomes nonlocal. Although material parameters still depend on wave characteristics in nonlocal theories, the latter provide better correspondence to the exact solutions, and therefore, their usage is justified for linear [24,30] and nonlinear media [31]. Nonlocality can be used for dispersion engineering [32,33] in transformation optics formalism applied to the spectral domain and for investigation of anisotropy induced by spatial dispersion [34]. When spatial dispersion is a shortcoming for some metamaterial-based application, one can apply the nonlocal homogenization approach to find out how to reduce undesired dispersion consequences. In the present paper we deal with nonlocal homogenization for a multilayered system.

Different materials forming the unit cell of a metamaterial can have a complex shape. However, it is instructive to use a simple toy model which can provide the main features of the metamaterial function. Such a simple model can be a periodic multilayered system, as demonstrated in Fig. 1(b). The homogenization in this case is given by the Maxwell Garnett (quasistatic) approach, which is called the effective medium approximation (EMA). Recently, the homogenization of the subwavelength dielectric layered structures has attracted close attention in connection with the discovered EMA breakdown [35-37]. The EMA breakdown typically is quite pronounced near the critical angle of total internal reflection defined for the effective medium. As reported in Ref. [35], the transmission of a multilayered structure strongly depends on periodicity, the order of the layers, and their parity, although such a dependence is supposed to be nonexistent when the EMA is applied, in particular for deep-subwavelength dielectric multilayers.

Metal-dielectric planar multilayered systems can be considered effectively anisotropic (uniaxial) media possessing either closed ellipsoid or open hyperboloid isofrequency surfaces. In the latter case the multilayers, called hyperbolic metamaterials, enable a number of distinctive properties, including guiding evanescent waves (with wave vectors much bigger than those in the corresponding uniform dielectric), hyperlensing, and enhancement of the spontaneous emission rate [10,38-42]. The EMA is applicable for multilayered hyperbolic metamaterials, too; however certain constraints must be acknowledged [43]. It should be noted that, typically, homogenization approaches have been developed for layered structures to catch some specific features. For example, the high-frequency homogenization is applicable near the points of topological transition from the elliptic to hyperbolic regime [44]. However, a general and consistent homogenization approach for multilayers is still missing.

In this paper, we use an operator technique to develop an operator effective medium approximation (OEMA), in which the zeroth order renders the conventional EMA (Maxwell Garnett theory), while higher orders ameliorate the accurateness of material tensors. The approach is grounded on the theory presented in Ref. [45], which is adapted to find the effective material tensors of the homogenized multilayered structure. In Sec. II we briefly discuss the propagation of plane waves in bianisotropic media using the spatial evolution operator technique developed in Refs. [46-48]. It was used, in particular, for analyses of planar waveguides modes [49] and retrieving bianisotropic material tensors from transmission and reflection spectra [50,51]. In Sec. III we outline the OEMA and show how to accurately determine material parameters of the effective medium. The zeroth-order OEMA for multilayered dielectric systems is considered in Sec. IV. This approach is limited to only electric dipoles. In Sec. V we discuss the first-order OEMA and its connection with the chiral properties of the multilayer. In the second-order OEMA, consequently, magnetic dipoles and electric quadrupoles are taken into account (Sec. VI). In Sec. VII we demonstrate that application of the second-order OEMA allows avoiding the EMA breakdown near the critical angle of total internal reflection. We also identify the criterion for the zeroth-order OEMA validity, which depends on the unit-cell thickness to wavelength ratio, the number of unit cells, and the angle of incidence. Section VIII advances application of the OEMA to frequencydispersive metal-dielectric multilayers. Comparison with the results of the latest high-frequency homogenization approach [44] and conclusions are also provided here.

\section{PLANE WAVES IN MULTILAYERED BIANISOTROPIC MEDIA}

We consider a plane monochromatic electromagnetic wave with circular frequency $\omega$ propagating in a bianisotropic slab characterized by dielectric permittivity tensor $\hat{\varepsilon}$, magnetic 
permeability tensor $\hat{\mu}$, and gyration pseudotensors $\hat{\alpha}$ and $\hat{\beta}[52]$,

$$
\begin{aligned}
& \mathbf{D}(\omega, \mathbf{r})=\hat{\varepsilon}(\omega) \mathbf{E}(\omega, \mathbf{r})+\hat{\alpha}(\omega) \mathbf{H}(\omega, \mathbf{r}), \\
& \mathbf{B}(\omega, \mathbf{r})=\hat{\beta}(\omega) \mathbf{E}(\omega, \mathbf{r})+\hat{\mu}(\omega) \mathbf{H}(\omega, \mathbf{r}) .
\end{aligned}
$$

Here $\mathbf{E}, \mathbf{H}, \mathbf{D}$, and $\mathbf{B}$ are, respectively, the strengths of electric and magnetic fields, electric displacement, and magnetic induction vectors. Under the oblique incidence the wave vector can be decomposed into two components, tangential $\mathbf{k}_{t}=k_{0} \mathbf{b}$ and longitudinal $\mathbf{k}_{z}=k_{0} \eta \mathbf{q}$, where $k_{0}=\omega / c$ is the wave number in vacuum, $\mathbf{b}$ lies in the plane of incidence, and $\mathbf{q}$ is the unit vector normal to the slab interfaces (see Fig. 1). Since the field is translation invariant in the plane $(x, y)$, it can be written in the form $\mathbf{E}(\mathbf{r})=\mathbf{E}(z) \exp \left(i k_{0} \mathbf{b} \cdot \mathbf{r}\right)$, thus reducing the Maxwell equations to the system of the four first-order ordinary differential equations for the tangential field components $\mathbf{W}=\left(\mathbf{H}_{t}, \mathbf{q} \times \mathbf{E}\right)^{T}$, where $T$ denotes the transpose operation. Here two tangential fields, $\mathbf{H}_{t}=\hat{I} \mathbf{H}=$ $\left(H_{x}, H_{y}\right)^{T}$ and $\mathbf{q} \times \mathbf{E}=\left(-E_{y}, E_{x}\right)^{T}$, are introduced, where $\hat{I}=\hat{1}-\mathbf{q} \otimes \mathbf{q}$ is the projector onto the plane orthogonal to $\mathbf{q}$ (two-dimensional unit tensor), $\hat{1}$ is the three-dimensional unit tensor (Kronecker's delta tensor), and $\otimes$ stands for the tensor (outer) product defined as $(\mathbf{u} \otimes \mathbf{v})_{i, j}=u_{i} v_{j}, i, j=1,2,3$.

The system of four differential equations can be rewritten as a single equation for unknown field vector $\mathbf{W}$ :

$$
\frac{d \mathbf{W}(z)}{d z}=i k_{0} \hat{M} \mathbf{W}(z)
$$

where $\hat{M}$ is the $4 \times 4$ matrix, whose representation as a block matrix reads [47]

$$
\hat{M}=\left(\begin{array}{ll}
\hat{A} & \hat{B} \\
\hat{C} & \hat{D}
\end{array}\right)
$$

In the case of bianisotropic media described by material equations (1), the $2 \times 2$ blocks $\hat{A}, \hat{B}, \hat{C}$, and $\hat{D}$ are

$$
\begin{aligned}
& \hat{A}=\mathbf{q}^{\times} \hat{\alpha} \hat{I}+\mathbf{q}^{\times} \hat{\varepsilon} \mathbf{q} \otimes \mathbf{v}_{3}+\left(\mathbf{b}+\mathbf{q}^{\times} \hat{\alpha} \mathbf{q}\right) \otimes \mathbf{v}_{1}, \\
& \hat{B}=-\mathbf{q}^{\times} \hat{\varepsilon} \mathbf{q}^{\times}+\mathbf{q}^{\times} \hat{\varepsilon} \mathbf{q} \otimes \mathbf{q}^{\times} \mathbf{v}_{4}+\left(\mathbf{b}+\mathbf{q}^{\times} \hat{\alpha} \mathbf{q}\right) \otimes \mathbf{q}^{\times} \mathbf{v}_{2}, \\
& \hat{C}=\hat{I} \hat{\mu} \hat{I}+\hat{I} \hat{\mu} \mathbf{q} \otimes \mathbf{v}_{1}+(-\mathbf{a}+\hat{I} \hat{\beta} \mathbf{q}) \otimes \mathbf{v}_{3}, \\
& \hat{D}=-\hat{I} \hat{\beta} \mathbf{q}^{\times}+\hat{I} \hat{\mu} \mathbf{q} \otimes \mathbf{q}^{\times} \mathbf{v}_{2}+(-\mathbf{a}+\hat{I} \hat{\beta} \mathbf{q}) \otimes \mathbf{q}^{\times} \mathbf{v}_{4},
\end{aligned}
$$

where $\mathbf{q}^{\times}$is the tensor dual to vector $\mathbf{q}[47,52,53]\left[\left(\mathbf{q}^{\times}\right)_{i k}=\right.$ $\varepsilon_{i j k} q_{j}$, where $\varepsilon_{i j k}$ is the antisymmetric Levi-Civita tensor and summation over repeated indices from 1 to 3 is assumed], $\mathbf{a}=\mathbf{b} \times \mathbf{q}$, and

$$
\begin{aligned}
\mathbf{v}_{1} & =\delta_{q}\left(\beta_{q} \mathbf{q} \hat{\alpha} \hat{I}-\varepsilon_{q} \mathbf{q} \hat{\mu} \hat{I}-\beta_{q} \mathbf{a}\right), \\
\mathbf{v}_{2} & =\delta_{q}\left(\beta_{q} \mathbf{q} \hat{\varepsilon} \hat{I}-\varepsilon_{q} \mathbf{q} \hat{\beta} \hat{I}-\varepsilon_{q} \mathbf{a}\right), \\
\mathbf{v}_{3} & =\delta_{q}\left(\alpha_{q} \mathbf{q} \hat{\mu} \hat{I}-\mu_{q} \mathbf{q} \hat{\alpha} \hat{I}+\mu_{q} \mathbf{a}\right), \\
\mathbf{v}_{4} & =\delta_{q}\left(\alpha_{q} \mathbf{q} \hat{\beta} \hat{I}-\mu_{q} \mathbf{q} \hat{\varepsilon} I+\alpha_{q} \mathbf{a}\right), \\
\delta_{q} & =\left(\varepsilon_{q} \mu_{q}-\alpha_{q} \beta_{q}\right)^{-1}, \quad \varepsilon_{q}=\mathbf{q} \hat{\varepsilon} \mathbf{q}, \\
\mu_{q} & =\mathbf{q} \hat{\mu} \mathbf{q}, \quad \alpha_{q}=\mathbf{q} \hat{\alpha} \mathbf{q}, \quad \beta_{q}=\mathbf{q} \hat{\beta} \mathbf{q} .
\end{aligned}
$$

In the case of a homogeneous bianisotropic medium matrix $\hat{M}$ is constant, thus allowing us to write down the fundamental solution of the system by means of the matrix exponential $[47,49]$

$$
\mathbf{W}(z)=\exp \left[i k_{0} z \hat{M}\right] \mathbf{W}(0),
$$

where $\mathbf{W}(0)$ is the initial field at $z=0$. The field transmitted by the first slab is the initial field for the second one, the second slab produces the initial field for the third slab at its output, and so on. Therefore, the field transmitted by the stack of $N$ slabs is of the form

$$
\mathbf{W}(D)=\prod_{j=1}^{N} \exp \left[i k_{0} d_{j} \hat{M}_{j}\right] \mathbf{W}(0)=\hat{P} \mathbf{W}(0),
$$

where $D=\sum_{j=1}^{N} d_{j}$ is the thickness of the stack. Tensor $\hat{P}$ can be called a spatial evolution operator because it describes the spatial evolution of the initial field $\mathbf{W}(0)$.

In Fig. 1(b) the periodic structure of $2 N$ alternating dielectric slabs with permittivities $\varepsilon_{1}$ and $\varepsilon_{2}$ and thicknesses $d_{1}$ and $d_{2}$ is considered. Oblique incidence of a plane wave in dielectric material $\varepsilon_{\text {in }}$ is assumed. The transmitted wave exits to the semi-infinite dielectric medium $\varepsilon_{\text {out }}$. For the parameters given in the caption of Fig. 2 the effective parameters in the zeroth-order OEMA are equal to $\varepsilon_{\|}^{(0)}=3$ and $\varepsilon_{\perp}^{(0)}=5 / 3$. Then the critical angle of total internal reflection for a TE wave is $\alpha_{c}=60^{\circ}$, which corresponds to the normalized tangential wave number $k_{t} / k_{0}=b_{c}=\sqrt{3}$.

Fresnel's (amplitude) transmission operator can also be written in terms of the spatial evolution operator $\hat{P}$ as [47]

$$
\left.\hat{t}=2\left[\begin{array}{ll}
\hat{\gamma}_{\text {in }} & \hat{I}
\end{array}\right) \hat{P}^{-1}\left(\begin{array}{c}
\hat{I} \\
\hat{\gamma}_{\text {out }}
\end{array}\right)\right]^{-1} \hat{\gamma}_{\text {in }},
$$

where $\hat{\gamma}_{\text {in }}$ and $\hat{\gamma}_{\text {out }}$ are the surface impedance tensors of the input and output media shown in Fig. 1(b). For instance, $\hat{\gamma}_{\text {in }}$ equals

$$
\hat{\gamma}_{\text {in }}=\frac{\sqrt{\varepsilon_{\text {in }}-b^{2}}}{b^{2} \varepsilon_{\text {in }}} \mathbf{a} \otimes \mathbf{a}+\frac{1}{b^{2} \sqrt{\varepsilon_{\text {in }}-b^{2}}} \mathbf{b} \otimes \mathbf{b} .
$$

The output magnetic field equals $\mathbf{H}_{t}^{(\mathrm{tr})}=\hat{t} \mathbf{H}_{t}^{(\mathrm{inc})}$, where $\mathbf{H}_{t}^{(\mathrm{inc})}$ is the magnetic field of the incident wave. Then the power transmission coefficient for a TE wave reads

$$
T=\frac{\operatorname{Re}\left[\eta_{\text {out }}^{-1}\right]\left|\mathbf{H}_{t}^{\text {(tr) }}\right|^{2}}{\operatorname{Re}\left[\eta_{\text {in }}^{-1}\right]\left|\mathbf{H}_{t}^{\text {(inc) }}\right|^{2}}
$$

where $\eta_{\text {in }}=\sqrt{\varepsilon_{\text {in }}-b^{2}}$ and $\eta_{\text {out }}=\sqrt{\varepsilon_{\text {out }}-b^{2}}$ are the longitudinal components of the input and output wave vectors normalized to $k_{0}$, respectively.

Fresnel's transmission coefficient $t$ for the TE-polarized wave passing through the $N$-period multilayer [see Fig. 1(b)] equals

$$
t=\left(\frac{\eta_{\text {in }}+\eta_{\text {out }}}{2 \eta_{\text {out }}} P_{2}+\frac{P_{1} P_{3}}{2 \eta_{\text {out }}}\right)^{-1},
$$



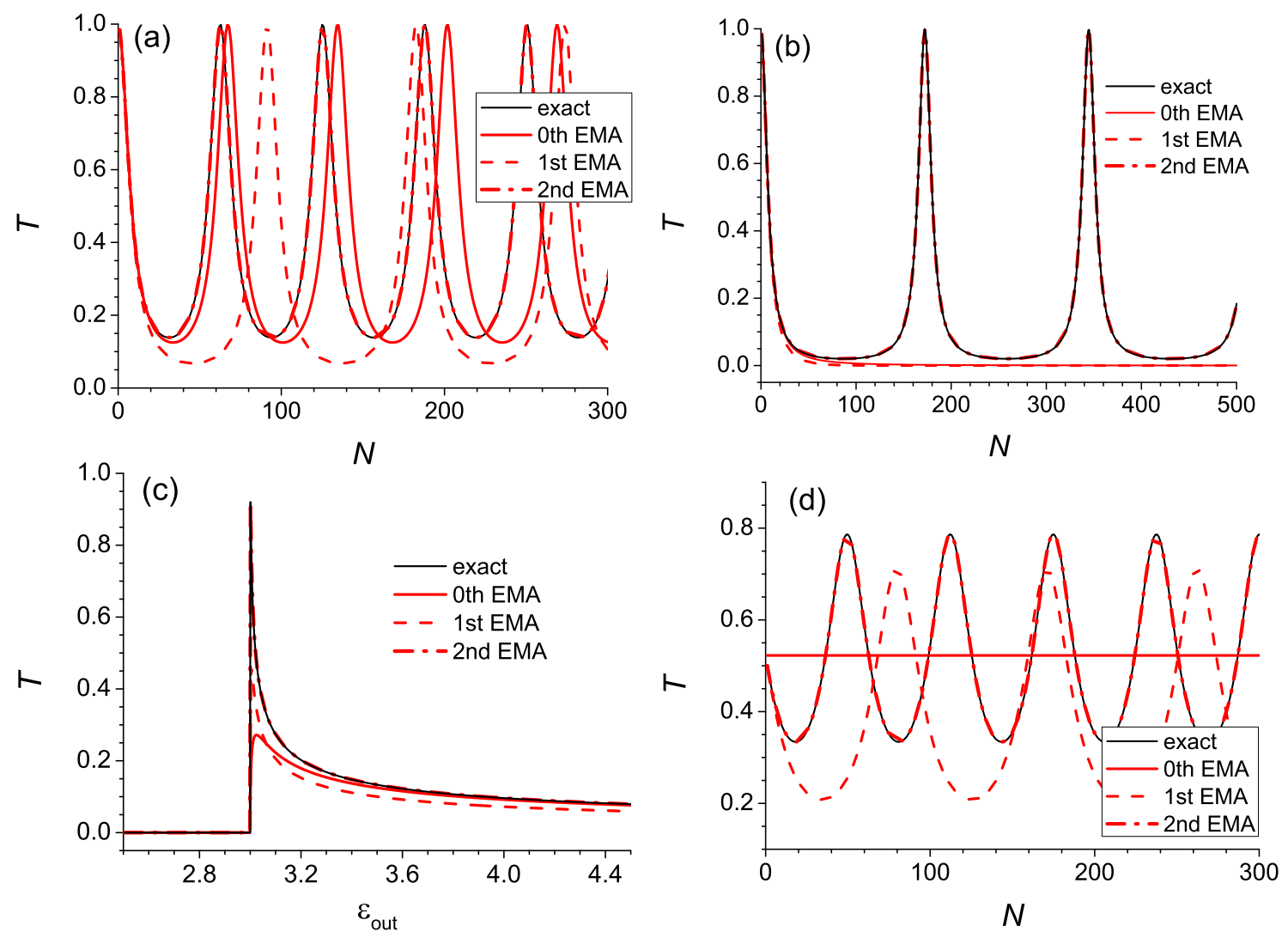

FIG. 2. Transmission of $s$ - (TE-) polarized light through the $N$-period stack of slabs calculated exactly, in the zeroth-order OEMA, in the first-order OEMA, and in the second order OEMA vs the number of unit cells $N$ for (a) subcritical $\left(\varepsilon_{\text {out }}=4, b=\sqrt{3}-0.01\right)$ and (b) critical angles of incidence $\left(\varepsilon_{\text {out }}=4, b=\sqrt{3}\right)$, (c) output permittivity $\varepsilon_{\text {out }}(N=25, b=\sqrt{3})$, and (d) the number of unit cells for the impedance-matched output medium $\left(\varepsilon_{\text {out }}=3, b=\sqrt{3}-0.01\right.$ ). Parameters are $\varepsilon_{1}=5, \varepsilon_{2}=1, d_{1}=d_{2}=10$ nm, $\varepsilon_{\text {in }}=4$, radiation wavelength $\lambda=500 \mathrm{~nm}$. The first layer of the stack is slab 1 in (a), (b), and (d) and slab 2 in (c).

where

$$
\begin{aligned}
& P_{1}=\frac{\sin \left(N K_{B} d\right)}{\sin \left(K_{B} d\right)}, \quad P_{2}=\cos \left(N K_{B} d\right), \\
& P_{3}=-P_{T E}^{12}-\eta_{\text {in }} \eta_{\text {out }} P_{T E}^{21}+\left(\eta_{\text {in }}-\eta_{\text {out }}\right) \frac{P_{T E}^{11}-P_{T E}^{22}}{2} .
\end{aligned}
$$

Here $d=d_{1}+d_{2}$ is the thickness of the unit cell. The dispersion equation for the Bloch wave number $K_{B}$ and elements $P_{T E}^{11}, P_{T E}^{12}, P_{T E}^{21}$, and $P_{T E}^{22}$ of the TE-wave evolution operator $\mathbf{b} \hat{P} \mathbf{b} / b^{2}$ are given in Appendix A.

\section{OPERATOR EFFECTIVE MEDIUM APPROXIMATION}

The EMA is usually derived using the Maxwell Garnett (quasistatic) approach. Then a homogeneous effective medium replacing the multilayered structure has quite simple material parameters of an anisotropic medium. The coordinate-free approach of such homogenization generalized for an arbitrary number of anisotropic layers defined through complex nonsymmetrical tensors $\hat{\varepsilon}, \hat{\mu}$ composing the unit cell are derived in [54]. In this section we apply an operator approach developed in Ref. [45] to improve the accuracy of the EMA. We will refer to this method as operator effective medium approximation.
The idea behind the OEMA is a conventional routine to replace the periodic structure of thickness $N d$ by a homogeneous slab (effective medium) of the same thickness $N d$, where $N$ and $d$ are the number of unit cells and thickness of a single unit cell, respectively. In general, the effective medium is bianisotropic, thus having dielectric $\hat{\varepsilon}_{\text {eff }}$ and magnetic $\hat{\mu}_{\text {eff }}$ tensors and gyration pseudotensors $\hat{\alpha}_{\text {eff }}$ and $\hat{\beta}_{\text {eff }}$ as material parameters. With the ideal homogenization we cannot distinguish the initial layered medium and effective medium because the electromagnetic field outside the homogeneous slab and inhomogeneous medium are the same. This can be achieved owing to the equality of the spatial evolution operators for homogeneous and inhomogeneous samples over a period:

$$
\exp \left[i k_{0} d \hat{M}_{\mathrm{eff}}\right]=\exp \left[i k_{0} d_{2} \hat{M}_{2}\right] \exp \left[i k_{0} d_{1} \hat{M}_{1}\right]
$$

It should be noted that the OEMA through the equality (11) is applicable even for a single period, i.e., for nonperiodic structures, too. Thus, in no way we are restricted by the number of periods $N$ in the process of homogenization. In the above discussion we assume an external illumination of the multilayer system. OEMA can also be applied when the light source is placed at the boundaries between periods, i.e., at $z=j d(j=1,2, \ldots, N-1)$. 
Matrix $\hat{M}_{\text {eff }}$ can be derived using the logarithm of the operator

$$
\hat{M}_{\text {eff }}=\frac{1}{i k_{0} d} \ln \left(\exp \left[i k_{0} d_{2} \hat{M}_{2}\right] \exp \left[i k_{0} d_{1} \hat{M}_{1}\right]\right)
$$

or through the Baker-Campbell-Hausdorff series [45]

$$
\begin{aligned}
\hat{M}_{\text {eff }}= & \rho \hat{M}_{1}+(1-\rho) \hat{M}_{2} \\
& +\frac{i k_{0} d}{2} \rho(1-\rho)\left[\hat{M}_{2}, \hat{M}_{1}\right] \\
& -\frac{\left(k_{0} d\right)^{2}}{12} \rho(1-\rho)\left(\rho\left[\left[\hat{M}_{2}, \hat{M}_{1}\right], \hat{M}_{1}\right]\right. \\
& \left.+(1-\rho)\left[\left[\hat{M}_{1}, \hat{M}_{2}\right], \hat{M}_{2}\right]\right)+\cdots,
\end{aligned}
$$

where $\rho=d_{1} / d$ and $\left[\hat{M}_{1}, \hat{M}_{2}\right]$ is a commutator. Equation (13) is the expansion with respect to the powers of $k_{0} d$. Since $M$ is the $4 \times 4$ matrix, Eq. (13) represents the system of 16 algebraic equations, which are not sufficient to uniquely determine 36 material parameters: each of the tensors $\hat{\varepsilon}_{\text {eff }}, \hat{\mu}_{\text {eff }}, \hat{\alpha}_{\text {eff }}$, and $\hat{\beta}_{\text {eff }}$ has, in general, nine components. Hence, there exists a group of equivalent effective media which are able to describe the same inhomogeneous structure.

It should be noted that such expansion over $k_{0} d$ was also used in other works [30,55]. In Ref. [55], the authors consider the zero-order term $\left(k_{0} d\right)^{0}$ corresponding to the local effective parameters and outline the way to introduce higher-order corrections to effective medium parameters. In Ref. [30], the expansion up to the second order is applied to the three-dimensional metamaterials to describe the effects of artificial chirality and spatial dispersion.

\section{ZEROTH-ORDER OEMA: ELECTRIC DIPOLES}

The Baker-Campbell-Hausdorff series (13) serves as the exact homogenization. If $k_{0} d$ is small, the series can be safely cut. The power of $k_{0} d$ defines the order of approximation: the $n$ th-order approximation corresponds to $\left(k_{0} d\right)^{n}$ in Eq. (13). Then the zeroth-order approximation of $\hat{M}_{\text {eff }}$ can be written as follows:

$$
\hat{M}_{\text {eff }}^{(0)}=\rho \hat{M}_{1}+(1-\rho) \hat{M}_{2} .
$$

If the wave and material parameters in $\hat{M}_{1,2}$ are not peculiar, the zeroth-order approximation is conventionally valid at $d \ll \lambda$, where $\lambda$ is the radiation wavelength.

Let us find material parameters from Eq. (14) in the case of alternating isotropic dielectric slabs with matrices $\hat{M}_{1}$ and $\hat{M}_{2}$. By substituting $\hat{\varepsilon}_{1,2}=\varepsilon_{1,2} \hat{1}, \hat{\mu}_{1,2}=\hat{1}, \hat{\alpha}_{1,2}=\hat{\beta}_{1,2}=0$ into Eq. (3), one obtains matrices $\hat{M}_{1,2}$ as

$$
\hat{M}_{1,2}=\left(\begin{array}{cc}
0 & \varepsilon_{1,2} \hat{I}-\mathbf{b} \otimes \mathbf{b} \\
\hat{I}-\frac{\mathbf{a} \otimes \mathbf{a}}{\varepsilon_{1,2}} & 0
\end{array}\right) .
$$

The effective medium can be regarded as a general bianisotropic one. Then its parameters appear not to be unique. However, the physical insight can significantly reduce the number of material parameters. In fact, the stack of isotropic slabs has a preferred direction specified by the vector $\mathbf{q}$. Then the effective medium material tensors can be assumed as $\hat{\varepsilon}_{\text {eff }}=\varepsilon_{\| \mid} \hat{I}+\varepsilon_{\perp} \mathbf{q} \otimes \mathbf{q}, \hat{\mu}_{\text {eff }}=\mu_{\| \mid} \hat{I}+\mu_{\perp} \mathbf{q} \otimes \mathbf{q}$, $\hat{\alpha}_{\text {eff }}=\alpha_{\|} \hat{I}+\alpha_{\perp} \mathbf{q} \otimes \mathbf{q}$, and $\hat{\beta}_{\text {eff }}=\beta_{\|} \hat{I}+\beta_{\perp} \mathbf{q} \otimes \mathbf{q}$. Matrix $\hat{M}$ in the zeroth-order EMA reads

$$
\hat{M}_{\text {eff }}^{(0)}=\left(\begin{array}{cc}
\alpha_{\| \mid} \mathbf{q}^{\times}-\frac{\beta_{\perp} \mathbf{b} \otimes \mathbf{a}}{\delta_{\perp}} & \varepsilon_{\|} \hat{I}-\frac{\varepsilon_{\perp} \mathbf{b} \otimes \mathbf{b}}{\delta_{\perp}} \\
\mu_{\|} \hat{I}-\frac{\mu_{\perp} \mathbf{a} \otimes \mathbf{a}}{\delta_{\perp}} & -\beta_{\|} \mathbf{q}^{\times}-\frac{\alpha_{\perp} \mathbf{a} \otimes \mathbf{b}}{\delta_{\perp}}
\end{array}\right),
$$

where $\delta_{\perp}=\varepsilon_{\perp} \mu_{\perp}-\alpha_{\perp} \beta_{\perp}$. By introducing matrices $\hat{M}$ into Eq. (14), we get a system of algebraic equations

$$
\begin{aligned}
\alpha_{\| \mid} \mathbf{q}^{\times} & -\left(\beta_{\perp} / \delta_{\perp}\right) \mathbf{b} \otimes \mathbf{a}=0, \\
\varepsilon_{\|} \hat{I} & -\left(\varepsilon_{\perp} / \delta_{\perp}\right) \mathbf{b} \otimes \mathbf{b}=\rho\left(\varepsilon_{1} \hat{I}-\mathbf{b} \otimes \mathbf{b}\right) \\
& +(1-\rho)\left(\varepsilon_{2} \hat{I}-\mathbf{b} \otimes \mathbf{b}\right), \\
\mu_{\|} \hat{I} & -\left(\mu_{\perp} / \delta_{\perp}\right) \mathbf{a} \otimes \mathbf{a}=\rho\left(\hat{I}-\mathbf{a} \otimes \mathbf{a} / \varepsilon_{1}\right) \\
& +(1-\rho)\left(\hat{I}-\mathbf{a} \otimes \mathbf{a} / \varepsilon_{2}\right), \\
& -\beta_{\|} \mathbf{q}^{\times}-\left(\alpha_{\perp} / \delta_{\perp}\right) \mathbf{a} \otimes \mathbf{b}=0 .
\end{aligned}
$$

The solution of this system of equations brings us to the nongyrotropic $\left(\hat{\alpha}_{\text {eff }}=\hat{\beta}_{\text {eff }}=0\right)$ nonmagnetic $\left(\hat{\mu}_{\text {eff }}=\hat{1}\right)$ uniaxial medium as the zeroth-order effective medium:

$$
\varepsilon_{\|}^{(0)}=\rho \varepsilon_{1}+(1-\rho) \varepsilon_{2}, \quad \varepsilon_{\perp}^{(0)}=\left(\frac{\rho}{\varepsilon_{1}}+\frac{1-\rho}{\varepsilon_{2}}\right)^{-1} .
$$

Thus, the zeroth-order material parameters are those obtained using the quasistatic Maxwell Garnett approach [40]. With the assumption of the form of the effective media tensors we determined them uniquely because eight unknown material parameters can be found from 16 equations of the system (14). It should be noted that the wrong ansatz of the effective medium tensors can result in the contradictory system of algebraic equations.

A dielectric multilayered system in the lowest order of the OEMA is just a dielectric described by the average electric dipole moment (medium polarization). Parameters (18) depend on neither the number of unit cells nor the location of the materials in the unit cells.

Fresnel's transmission coefficient of the multilayered structure in the zeroth-order approximation $t^{(0)}$ can be written in a way similar to Eq. (9), but the quantities $P$ are replaced by $P^{(0)}$ :

$$
\begin{aligned}
& P_{1}^{(0)}=\frac{\sin \left(N k_{0} \eta^{(0)} d\right)}{\sin \left(k_{0} \eta^{(0)} d\right)}, \quad P_{2}^{(0)}=\cos \left(N k_{0} \eta^{(0)} d\right), \\
& P_{3}^{(0)}=\frac{i \sin \left(k_{0} \eta^{(0)} d\right)}{\eta^{(0)}}\left[-\left(\eta^{(0)}\right)^{2}-\eta_{\text {in }} \eta_{\text {out }}\right],
\end{aligned}
$$

where $\eta^{(0)}=\sqrt{\varepsilon_{\|}^{(0)}-b^{2}}$ is the longitudinal component of the wave vector in the zeroth- order OEMA normalized to $k_{0}$.

Despite the validity condition of EMA $d=d_{1}+d_{2} \ll$ $\lambda$, the zeroth-order approximation can fail when either the number of unit cells exceeds a certain value [see Figs. 2(a) and 2(b)] or the ambient medium is impedance matched [see the example in Figs. 2(c) and 2(d)].

\section{FIRST-ORDER OEMA: CHIRALITY}

Matrix $\hat{M}$ in the first-order OEMA

$\hat{M}_{\text {eff }}^{(1)}=\rho \hat{M}_{1}+(1-\rho) \hat{M}_{2}+\frac{i k_{0} d}{2} \rho(1-\rho)\left[\hat{M}_{2}, \hat{M}_{1}\right]$ 
breaks the symmetry with respect to the sequence of the slabs. Starting the stack from layer 2 one exchanges $\hat{M}_{1}$ and $\hat{M}_{2}$ and therefore changes the sign of the last term.

Since the commutator

$$
\frac{i k_{0} d}{2} \rho(1-\rho)\left[\hat{M}_{2}, \hat{M}_{1}\right]=\left(\begin{array}{cc}
\hat{A}_{0} & 0 \\
0 & -\hat{A}_{0}
\end{array}\right)
$$

is a matrix with nonzero diagonal blocks, the effective medium matrix $\hat{M}_{\text {eff }}^{(1)}$ can have nonzero gyration pseudotensors $\hat{\alpha}_{\text {eff }}$ and $\hat{\beta}_{\text {eff }}$. Thus, the chiral properties of the effective material are built up only in the first-order approximation and therefore are quite small as $k_{0} d \ll 1$. Keeping $\hat{\mu}_{\text {eff }}=\hat{1}$ and a permittivity tensor as in Eq. (18), we shall find the gyration pseudotensors. Constant $\hat{\varepsilon}_{\text {eff }}$ and $\hat{\mu}_{\text {eff }}$ require invariant $\hat{B}$ and $\hat{C}$ in Eq. (3), that is, $\mathbf{q} \hat{\alpha}_{\text {eff }} \hat{I}=\mathbf{q} \hat{\beta}_{\text {eff }} \hat{I}=\hat{I} \alpha_{\text {eff }} \mathbf{q}=\hat{I} \beta_{\text {eff }} \mathbf{q}=0$. Imposing conditions $\alpha_{q}=\beta_{q}=0$, one derives the blocks of matrix $\hat{M}_{\text {eff }}: \hat{A}=\mathbf{q}^{\times} \hat{\alpha}_{\text {eff }} \hat{I}$ and $\hat{D}=-\hat{I} \hat{\beta}_{\text {eff }} \mathbf{q}^{\times}$. Finally, we derive the gyration pseudotensors as

$$
\hat{\alpha}_{\mathrm{eff}}=-\hat{\beta}_{\mathrm{eff}}^{T}=\hat{I} \hat{\alpha}_{\mathrm{eff}} \hat{I}=-\mathbf{q}^{\times} \hat{A}_{0}=\alpha_{1} \frac{\mathbf{a} \otimes \mathbf{b}}{b^{2}}+\alpha_{2} \frac{\mathbf{b} \otimes \mathbf{a}}{b^{2}},
$$

where

$$
\begin{aligned}
\alpha_{1} & =\frac{i k_{0} d}{2} \sigma, \quad \alpha_{2}=\frac{i k_{0} d}{2} \sigma f(b), \quad f(b)=\frac{b^{2}}{\varepsilon_{r}}-1, \\
\sigma & =\rho(1-\rho)\left(\varepsilon_{2}-\varepsilon_{1}\right) .
\end{aligned}
$$

Here $\varepsilon_{r}=\left(\varepsilon_{1}^{-1}+\varepsilon_{2}^{-1}\right)^{-1}$ is the reduced permittivity. When the stack starts from layer $2(1 \leftrightarrow 2), \hat{\alpha}_{\text {eff }}$ and $\hat{\beta}_{\text {eff }}$ change their signs.

Eigenwaves of the effective medium of the first order are TE- and TM-polarized plane waves. Although the obtained constitutive relations are very similar to those for uniaxial $\Omega$ structures [56], the coupling between the electric and magnetic fields is different for TE and TM waves, i.e., $\alpha_{1} \neq \alpha_{2}$. It is curious that this result is in contrast to that obtained in Ref. [30], where it was shown that the structure under consideration is a genuine uniaxial $\Omega$ medium.

It should be noted that the effective gyrotropy is surface induced because it can be removed by transferring half of the last slab to the place before the first layer, which makes the structure symmetric. Volume gyrotropy, in principle, can also be achieved, e.g., using a three-layer unit cell without an inversion center.

Parameters $P_{j}^{(1)}$ in the transmission coefficients (9) of the TE-polarized wave in the first-order approximation are equal to

$$
\begin{aligned}
& P_{1}^{(1)}=\frac{\sin \left(N k_{0} \eta^{(1)} d\right)}{\sin \left(k_{0} \eta^{(1)} d\right)}, \quad P_{2}^{(1)}=\cos \left(N k_{0} \eta^{(1)} d\right), \\
& P_{3}^{(1)}=\frac{i \sin \left(k_{0} \eta^{(1)} d\right)}{\eta^{(1)}}\left[-\left(\eta^{(0)}\right)^{2}-\eta_{\text {in }} \eta_{\text {out }}+\left(\eta_{\text {in }}-\eta_{\text {out }}\right) \alpha_{1}\right],
\end{aligned}
$$

where $\eta^{(1)}=\sqrt{\varepsilon_{\|}^{(0)}-b^{2}+\alpha_{1}^{2}}$ is the normalized longitudinal wave number of the wave. The dependence of $\eta^{(1)}$ versus $b$ is the dispersion equation of the effective medium in the first-order approximation.
The first two approximations of OEMA work well only for a small number of unit cells $N$ [Fig. 2(a)]. Further, both approximations fail, with the zeroth-order OEMA being even closer to the exact solution than the first order, when $\varepsilon_{\text {in }}=\varepsilon_{\text {out }}$, i.e., when chirality owing to the same ambient media does not appear.

The obtained first-order correction for the material parameters results in, however, the second-order corrections for the wave number of the plane wave in a homogenized medium because the Fresnel equation gets the correction $\alpha_{1}^{2}$ or $\alpha_{2}^{2}$ depending on the wave polarization. So the spatially dispersive chiral parameters should result in the second-order effect, and the second-order OEMA should be used for more accurate prediction of the reflection and transmission.

It should be noted that, alternatively, chiral material parameters can be assigned with a Hermitian permittivity tensor of the form $\hat{\varepsilon}_{\text {eff }}=\hat{\varepsilon}_{\text {sym }}+i \mathbf{h}^{\times}$, where $\hat{\varepsilon}_{\text {sym }}=\hat{\varepsilon}_{\text {sym }}^{T}$ and $\mathbf{h}$ is a nonunitary vector, while $\alpha_{\text {eff }}=\beta_{\text {eff }}=0$. Such a form of $\hat{\varepsilon}$ is often used to describe the Faraday optical activity induced by the magnetic field [52].

\section{SECOND-ORDER OEMA: MAGNETIC DIPOLES AND ELECTRIC QUADRUPOLES}

Material tensors of the second-order OEMA can be searched like those of a bianisotropic crystal. Since the third term in Eq. (13) maintains when the sequence of the slabs changes to the opposite one, the second order does not affect the chiral properties. The gyration pseudotensors can be kept as in Eq. (22), but the permittivity and permeability tensors have to be changed to incorporate proportional to $\left(k_{0} d\right)^{2}$ terms:

$$
\begin{aligned}
& \varepsilon_{\|}=\varepsilon_{\|}^{(0)}+\frac{\left(k_{0} d\right)^{2}}{6} \sigma f(b) \tilde{\varepsilon}_{\|}, \\
& \varepsilon_{\perp}=\varepsilon_{\perp}^{(0)}-\frac{\left(k_{0} d\right)^{2}}{6} \sigma \varepsilon_{\perp}^{(0) 2}\left(\frac{2 \rho-1}{\varepsilon_{r}}-\frac{f(b)}{\tilde{\varepsilon}_{\perp}}\right), \\
& \mu_{\|}=1+\frac{\left(k_{0} d\right)^{2}}{6} \sigma(2 \rho-1), \\
& \mu_{\perp}=1-\frac{\left(k_{0} d\right)^{2}}{6} \sigma\left(\rho \frac{\varepsilon_{1}}{\varepsilon_{2}}-(1-\rho) \frac{\varepsilon_{2}}{\varepsilon_{1}}\right),
\end{aligned}
$$

where

$$
\tilde{\varepsilon}_{||}=\rho \varepsilon_{1}-(1-\rho) \varepsilon_{2}, \quad \tilde{\varepsilon}_{\perp}=\left(\frac{\rho}{\varepsilon_{1}}-\frac{1-\rho}{\varepsilon_{2}}\right)^{-1} .
$$

Corrections introduced in this section depend on the tangential wave number $k_{t}=b k_{0}$; hence, the spatial dispersion is taken into account. The spatial dispersion effect manifests that the structure is thick compared with the radiation wavelength. One expects that the higher-order multipole moments besides the electric dipole will be excited. In fact, an artificial magnetic response is observed in Eq. (25), and it is not spatially dispersive. The correction to the dielectric permittivity tensor originates from the electric quadrupole moment, which depends on the tangential wave number introducing a spatial dispersion. Values of the magnetic dipole and electric quadrupole moments are of the same order, as anticipated for a nonmagnetic object. 
The eigenwaves in the second-order OEMA are TE- and TM-polarized plane waves like in the first-order OEMA, while the medium can be treated as a uniaxial $\Omega$ medium with different coupling constants $\alpha_{1,2}$.

The second-order OEMA yields the following parameters $P_{j}$ to substitute into Eq. (9) for TE-polarized light:

$$
\begin{aligned}
P_{1}^{(2)}= & \frac{\sin \left(N k_{0} \eta^{(2)} d\right)}{\sin \left(k_{0} \eta^{(2)} d\right)}, \quad P_{2}^{(2)}=\cos \left(N k_{0} \eta^{(2)} d\right), \\
P_{3}^{(2)}= & \frac{i \mu_{\|} \sin \left(k_{0} \eta^{(2)} d\right)}{\eta^{(2)}}\left[\left(\eta_{\text {in }}-\eta_{\text {out }}\right) \frac{\alpha_{1}}{\mu_{\|}}-\eta_{\text {in }} \eta_{\text {out }}\right. \\
& \left.+\frac{\alpha_{1}^{2}-\left(\eta^{(2)}\right)^{2}}{\mu_{\|}^{2}}\right],
\end{aligned}
$$

where the dimensionless longitudinal wave number equals $\eta^{(2)}=\sqrt{\alpha_{1}^{2}+\varepsilon_{\|} \mu_{\|}-\mu_{\|} / \mu_{\perp} b^{2}}$.

\section{SECOND-ORDER OEMA WITHOUT A BREAKDOWN}

As shown in Ref. [35], the periodic multilayered structure, parameters of which are indicated in the caption of Fig. 2, violates the main predictions of the effective medium approximation (zeroth-order OEMA in this paper). We shall demonstrate that the elaborated corrections to the material parameters solve the problem of the EMA breakdown.

For the subcritical angle of incidence the zeroth- and firstorder OEMAs keep the periodic dependencies characteristic to the exact solution but quantitatively differ, as shown in Fig. 2(a). Taking into consideration the effects of the structure's chirality, magnetic dipole, and electric quadrupole moments, we restore the correct behavior of transmission $T$ for any number of unit cells $N$. Moving to the critical angle, the transmission in the zeroth- and first-order OEMAs changes drastically [see Fig. 2(b)]. In this case, the zeroth-order effective medium does not transmit electromagnetic waves in accordance with the phenomenon of the total internal reflection except for the range of small $N$ when the waves instead tunnel through the thin structure. These tunneling waves result in the transmission corresponding to that calculated accurately.

In Fig. 2(c) we show the transmission at the critical angle (i.e., $b=\sqrt{3}$ ) as a function of the permittivity $\varepsilon_{\text {out }}$ of the output medium. The effective permittivity of the multilayer is $\varepsilon_{\|}^{(0)}=3$. When $\varepsilon_{\text {out }} \leqslant 3$, the effective permittivity of the system "periodic structure and output medium" is not greater than 3. Hence, the total internal reflection occurs, and $T=0$. For $\varepsilon_{\text {out }}>3$ the system is transmissive with the greatest value at $\varepsilon_{\text {out }} \approx \varepsilon_{\|}^{(0)}$. The sharp peak in Fig. 2(c) that cannot be caught by the zeroth-order OEMA is reproduced by the second-order OEMA even though the second-order corrections of material parameters are small by default and have no peculiarities with respect to $b$. This means that the resonance exists only in transmission near the critical angle and is extremely sensitive to the parameters of the system. Transmission in the peculiar case of the impedance-matched output medium $\left(\varepsilon_{\text {out }}=\varepsilon_{\|}^{(0)}\right)$ is shown in Fig. 2(d). It should be noticed that the behavior of $T$ is totally different for the exact solution and in the zerothorder OEMA. $T$ is constant in the zeroth-order OEMA because the multilayer and output substrate are effectively impedance matched and no dependence on $N$ is detected. The secondorder OEMA still functions perfectly.

In order to find out the reasons for the EMA breakdown we analyze the transmission coefficient $T$ for the incident TE-polarized electromagnetic wave. Fresnel's transmission coefficient of the multilayered structure composed of $N$ layer pairs is given by Eq. (9). It should be compared with the transmission coefficient $t^{(0)}$ of the zeroth-order effective medium of thickness $N d$, which follows from Eq. (9) when $P=P^{(0)}$ according to Eq. (19).

The difference between coefficients $t$ and $t^{(0)}$ is apparently caused by the discrepancy in coefficients $P_{j}$ and $P_{j}^{(0)}(j=$ $1,2,3)$. Since we consider subwavelength structures, it is instructive for further analysis to use approximation $k_{0} d \ll 1$. We can write the difference in transmission $\Delta T=T-T^{(0)}$ as a linear function of deviations $\delta P_{j}=P_{j}-P_{j}^{(0)}$ (see Appendix $\mathrm{B}$ for details):

$$
\begin{aligned}
\Delta T= & -2 T^{(0)} \operatorname{Re}\left(t^{(0)} \frac{\eta_{\text {in }}+\eta_{\text {out }}}{2 \eta_{\text {out }}} \delta P_{2}\right. \\
& \left.+\frac{t^{(0)}}{2 \eta_{\text {out }}}\left(P_{3}^{(0)} \delta P_{1}+P_{1}^{(0)} \delta P_{3}\right)\right) .
\end{aligned}
$$

If the terms on the right-hand side are small, the zeroth-order OEMA works well. Otherwise, the zeroth-order OEMA cannot be applied, and the phenomenon is called the EMA breakdown [35]. We aim to derive the conditions of the breakdown.

Each of the quantities in the transmission difference (27) is calculated in Appendix B, establishing the dependence on $k_{0} d, N$, and $\eta$. By substituting the values of $P_{j}^{(0)}$ and $\delta P_{j}$ into Eq. (27) in approximation $k_{0} d \ll 1$, we have

$$
\Delta T=A\left(\eta_{\text {out }}-\eta_{\text {in }}\right) \sigma\left(k_{0} d\right)+\mathrm{O}\left[\left(k_{0} d\right)^{2}\right]
$$

for $\eta^{(0)} \neq 0$ and

$$
\Delta T=N\left\{B\left(\eta_{\text {out }}-\eta_{\text {in }}\right) \sigma\left(k_{0} d\right)^{2}+\mathrm{O}\left[\left(k_{0} d\right)^{3}\right]\right\}
$$

for $\eta^{(0)} \rightarrow 0$, where $A$ and $B$ are the coefficients derived in Appendix B. The term proportional to $N\left(k_{0} d\right)^{3}$ arises due to the wave-number deviation $\Delta K d=\left(K_{B}-k_{0} \eta^{(0)}\right) d \sim\left(k_{0} d\right)^{3}$ (see Appendix A). It is less than other terms stemming from chirality and spatial dispersion. In the case $\eta^{(0)} \neq 0$ the transmission difference linearly depends on $k_{0} d$ as it is commonly adopted. But when $\eta^{(0)} \rightarrow 0$, the transmission difference grows with the number of unit cells $N$, yielding the great deviation, in accordance with Fig. 2(a).

If $\varepsilon_{\text {in }}=\varepsilon_{\text {out }}$ (or $\eta_{\text {in }}=\eta_{\text {out }}$ ), the term proportional to $\sigma$ in the deviation $\delta P_{3}$ vanishes (see Appendix B). This means that $\delta P_{3} \sim\left(k_{0} d\right)^{3}$ and the EMA works better. In fact, the transmission difference is proportional to $\Delta T \sim\left(k_{0} d\right)^{2}$ for $\eta^{(0)} \neq 0$ and $\Delta T \sim N\left(k_{0} d\right)^{3}$ for $\eta^{(0)} \rightarrow 0$.

Thus, the criterion for the zeroth-order OEMA to be valid is no longer $k_{0} d \ll 1$ but

$$
\frac{\sin \left[N \eta^{(0)} k_{0} d\right]\left(k_{0} d\right)}{\eta^{(0)} \eta_{\text {out }}} \ll 1
$$

for $\varepsilon_{\text {in }} \neq \varepsilon_{\text {out }}$ and

$$
\frac{\sin \left[N \eta^{(0)} k_{0} d\right]\left(k_{0} d\right)^{2}}{\eta^{(0)} \eta_{\text {out }}} \ll 1
$$

for $\varepsilon_{\text {in }}=\varepsilon_{\text {out }}$. 
In the first-order OEMA $\eta^{(1)}$ differs from $\eta^{(0)}$ by the term proportional to $\left(k_{0} d\right)^{2}$. Therefore, $\Delta K d \sim\left(k_{0} d\right)^{3}$ is not more precise than that in the zeroth-order OEMA. At the same time $\delta P_{3} \sim\left(k_{0} d\right)^{3}$, whose estimation is more accurate than in the zeroth order for $\varepsilon_{\text {in }} \neq \varepsilon_{\text {out }}$. Thus, the first-order OEMA is better than the zeroth one only if $\varepsilon_{\text {in }} \neq \varepsilon_{\text {out }}$. Figure 2(d) supports our conclusion: the first-order OEMA has oscillating behavior as the exact solution, while the zeroth-order approximation exhibits a constant value.

In the second-order OEMA $\Delta K d \sim\left(k_{0} d\right)^{5}$ and $\delta P_{3} \sim$ $\left(k_{0} d\right)^{5}$ because the terms proportional to $\left(k_{0} d\right)^{3}$ cancel each other. Then there is no peculiarity of the transmission at $\eta^{(0)}=$ 0 and $\Delta T \sim N\left(k_{0} d\right)^{5}$. This approximation can be violated if $N^{*} \Delta K d \sim 1$, i.e., $N^{*} \sim\left(k_{0} d\right)^{-5}$. For the parameters in Fig. 2 we estimate $N^{*} \sim 1000$

\section{DISCUSSION AND CONCLUSION}

The second-order OEMA is applicable within an extremely wide range of multilayered nanostructures. In what follows we show that it can describe wave propagation in frequencydispersive media as well. We compare the transmission in the zeroth-, first-, and second-order OEMAs with those calculated accurately and using the high-frequency homogenization [44]. In the stack of metal-dielectric slabs, only metal is assumed to have the Drude-model dispersion. When the validity conditions of the ordinary EMA hold true [see Fig. 3(a)], the high-frequency homogenization and zeroth-order OEMA are very close but differ heavily from the exact solution. The latter is indistinguishable from the second-order EMA, although the first-order OEMA is insufficient. When thickness $d$ is not much less than the wavelength [see Fig. 3(b)], the second-order EMA is not a valid approximation anymore. Nevertheless, it catches the resonances of the exact solution, thus exhibiting similar behavior. Other homogenization models considered cannot be used at all outside their applicability ranges.

The operator effective medium approximation is a general method to homogenize an arbitrary multilayered system. It is valid for a small number of slabs in the system, e.g., two slabs, as well as for any periodic and nonperiodic structure. The slabs of these structures can be anisotropic, magnetic, and chiral. OEMA does not require the development of a special technique for another composition of layers. The method is straightforward, but the technical difficulties should be solved to succeed in finding material tensors of the effective medium.

Although the idea of the OEMA is given in Ref. [45], we have analyzed different orders of the effective medium approximation for bilayer unit cells in detail. We have revealed that the orders of the approximation correspond to the appearance of the artificial multipole moments in the structure, with the value $k_{0} d$ being the dimensionless size parameter as in scattering theory [57]. When $k_{0} d$ is small, the electric dipole moment is important. Greater $k_{0} d$ produces higherorder artificial multipole moments. We apply the second-order OEMA to avoid the EMA breakdown in the vicinity of the critical angle of the total internal reflection found earlier [35]. The breakdown in the zeroth-order OEMA is unavoidable because transmission near the critical angle is very sensitive to the exact values of the effective parameters: even a very small
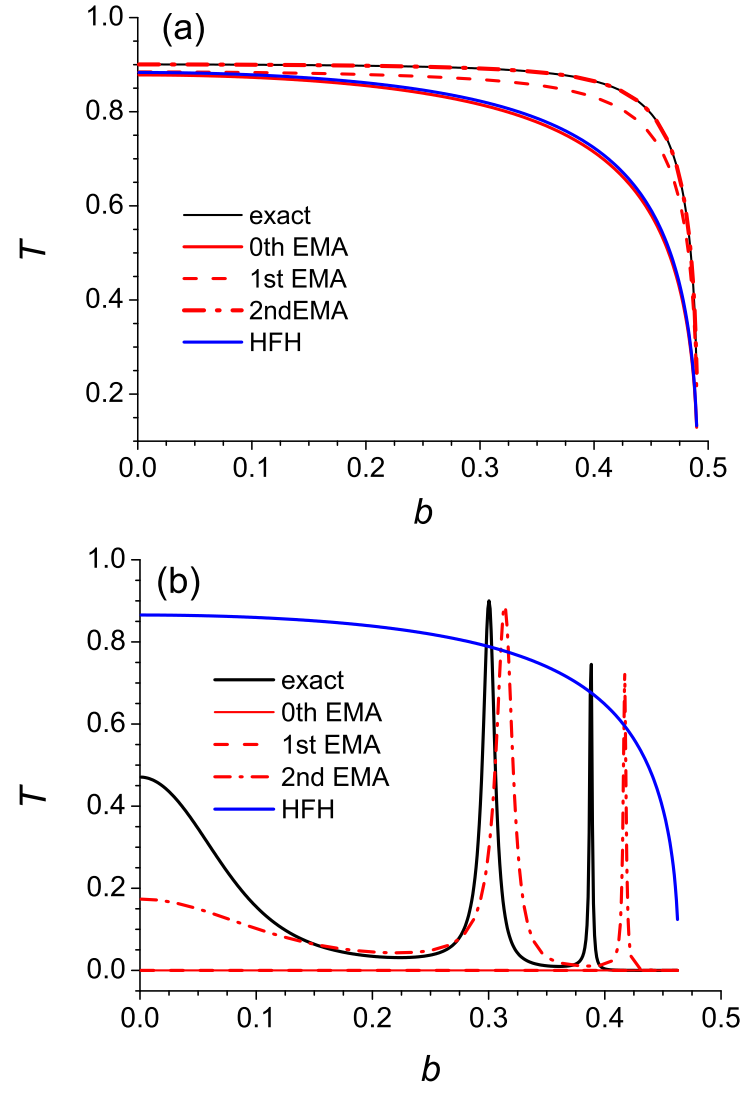

FIG. 3. Transmission of $s$ - (TE-) polarized light through the metal-dielectric structure calculated exactly, in the zeroth-order OEMA, in the first-order OEMA, in the second-order OEMA, and with the high-frequency homogenization $(\mathrm{HFH})$ approach vs normalized tangential wave number $b=k_{t} / k_{0}$ for (a) $k_{0} d=0.24\left(d_{1}=\right.$ $\left.d_{2}=0.2 c / \omega_{p}, \omega=0.6 \omega_{p}, \varepsilon_{\text {in }}=1, \varepsilon_{\text {out }}=0.241\right)$ and (b) $k_{0} d=2$ $\left(d_{1}=d_{2}=2 c / \omega_{p}, \omega=0.5 \omega_{p}, \varepsilon_{\text {in }}=1, \varepsilon_{\text {out }}=0.215\right)$. Parameters are $\varepsilon_{1}=1-\omega_{p}^{2} / \omega^{2}, \varepsilon_{2}=2.25, N=10$.

correction of the material parameters dramatically changes transmission.

In conclusion, we have found the effective medium tensors in different orders of the operator effective medium approximation. Each order corresponds to the artificial multipole moments. We have revealed that the second-order OEMA accurately describes the periodic multilayered structure even in such peculiar conditions as total internal reflection and a dispersive hyperbolic metamaterial. OEMA in the zeroth-order approximation can be used to find local parameters of a multilayered structure composed of anisotropic slabs.

\section{ACKNOWLEDGMENTS}

We are grateful to P. Belov, S. Maslovski, and the anonymous reviewer for fruitful discussions. We acknowledge the Belarusian Republican Foundation for Fundamental Research (Grant No. F16R-049) for financial support. Partial financial support from the Villum Fonden via the DarkSILD project is acknowledged. 


\section{APPENDIX A: COEFFICIENTS $P_{T E}^{11}, P_{T E}^{12}, P_{T E}^{21}$, AND $P_{T E}^{22}$ AND BLOCH WAVE NUMBER $K_{B}$}

The evolution operator $\hat{P}$ of a bilayer unit cell for a TEpolarized electromagnetic wave with fields $(\mathbf{b} / b) \cdot \mathbf{W}=(\mathbf{b}$. $\left.\mathbf{H}_{t} / b, \mathbf{a} \cdot \mathbf{E}_{t} / b\right)^{T}$ should be projected onto unit vector $\mathbf{b} / b$ as

$$
\hat{P}_{T E}=\frac{\mathbf{b} \hat{P} \mathbf{b}}{b^{2}}=\left(\begin{array}{cc}
P_{T E}^{11} & P_{T E}^{12} \\
P_{T E}^{21} & P_{T E}^{22}
\end{array}\right) .
$$

The elements of $\hat{P}_{T E}$ are of the form

$$
\begin{aligned}
P_{T E}^{11}= & \cos \left(k_{z 1} d_{1}\right) \cos \left(k_{z 2} d_{2}\right)-\frac{k_{z 2}}{k_{z 1}} \sin \left(k_{z 1} d_{1}\right) \sin \left(k_{z 2} d_{2}\right), \\
P_{T E}^{12}= & \frac{i}{k_{0}}\left[\sin \left(k_{z 2} d_{2}\right) \cos \left(k_{z 1} d_{1}\right) k_{z 2}+\sin \left(k_{z 1} d_{1}\right)\right. \\
& \left.\times \cos \left(k_{z 2} d_{2}\right) k_{z 1}\right], \\
P_{T E}^{21}= & i k_{0}\left[\sin \left(k_{z 2} d_{2}\right) \cos \left(k_{z 1} d_{1}\right) / k_{z 2}+\sin \left(k_{z 1} d_{1}\right)\right. \\
& \left.\times \cos \left(k_{z 2} d_{2}\right) / k_{z 1}\right], \\
P_{T E}^{22}= & \cos \left(k_{z 1} d_{1}\right) \cos \left(k_{z 2} d_{2}\right)-\frac{k_{z 1}}{k_{z 2}} \sin \left(k_{z 1} d_{1}\right) \sin \left(k_{z 2} d_{2}\right),
\end{aligned}
$$

where $k_{j z}=k_{0} \sqrt{\varepsilon_{j}-b^{2}}(j=1,2)$ is the longitudinal component of the wave vector in the $j$ th layer.

When $k_{0} d \ll 1$, one obtains

$$
\begin{aligned}
P_{T E}^{11} & =1-\frac{k_{z 1}^{2} d_{1}^{2}+k_{z 2}^{2} d_{2}^{2}}{2}-k_{z 2}^{2} d_{1} d_{2}+\mathrm{O}\left[\left(k_{0} d\right)^{4}\right], \\
P_{T E}^{12} & =\frac{i}{k_{0}}\left(k_{z 1}^{2} d_{1}+k_{z 2}^{2} d_{2}\right)+\mathrm{O}\left[\left(k_{0} d\right)^{3}\right], \\
P_{T E}^{21} & =i k_{0}\left(d_{1}+d_{2}\right)+\mathrm{O}\left[\left(k_{0} d\right)^{3}\right], \\
P_{T E}^{22} & =1-\frac{k_{z 1}^{2} d_{1}^{2}+k_{z 2}^{2} d_{2}^{2}}{2}-k_{z 2}^{2} d_{1} d_{2}+\mathrm{O}\left[\left(k_{0} d\right)^{4}\right] .
\end{aligned}
$$

The Bloch wave number $K_{B}$ follows from the dispersion equation

$$
\cos \left[K_{B} d\right]=\frac{P_{T E}^{11}+P_{T E}^{22}}{2} .
$$

For $k_{0} d \ll 1$ this equation reduces to

$$
\cos \left[K_{B} d\right]=1-\frac{\eta^{(0) 2} k_{0}^{2} d^{2}}{2}+\mathrm{O}\left[\left(k_{0} d\right)^{4}\right],
$$

whose solution is $K_{B} d=k_{0} \eta^{(0)} d+\mathrm{O}\left[\left(k_{0} d\right)^{3}\right]$. Therefore, the wave-number difference equals $\Delta K d=K_{B} d-k_{0} \eta^{(0)} d=$ $\mathrm{O}\left[\left(k_{0} d\right)^{3}\right]$.

\section{APPENDIX B: TRANSMISSION COEFFICIENT DIFFERENCE}

Fresnel's transmission coefficient (9) can be written as a linear function of the deviations from the zeroth-order approximation $\delta P_{j}=P_{j}-P_{j}^{(0)}(j=1,2,3)$, if $\left|\delta P_{j}\right| \ll\left|P_{j}\right|$ :

$$
t=t_{0}\left(1-t_{0} \tau\right)
$$

where

$$
\tau=\frac{\eta_{\text {in }}+\eta_{\text {out }}}{2 \eta_{\text {out }}} \delta P_{2}+\frac{\delta P_{1} P_{3}^{(0)}+P_{1}^{(0)} \delta P_{3}}{2 \eta_{\text {out }}}
$$

Then the transmission difference $\Delta T=T-T^{(0)}$ takes the form

$$
\Delta T=\frac{\eta_{\text {in }}}{\eta_{\text {out }}}|t|^{2}-\frac{\eta_{\text {in }}}{\eta_{\text {out }}}\left|t^{(0)}\right|^{2}=-2 T^{(0)} \operatorname{Re}\left(t_{0} \tau\right) .
$$

$\Delta T$ in the explicit form is demonstrated by Eq. (27).

Let us calculate $P_{j}^{(0)}$ and $\delta P_{j}$ in approximation $k_{0} d \ll 1$. In the expressions we can treat $\sin \left(k_{0} \eta^{(0)} d\right) \approx k_{0} \eta^{(0)} d$, but $N k_{0} \eta^{(0)} d$ is large, and $\sin \left(N k_{0} \eta^{(0)} d\right) \neq N k_{0} \eta^{(0)} d$ except in the case of $\eta^{(0)} \rightarrow 0$.

Then

$$
\begin{aligned}
& P_{1}^{(0)}= \begin{cases}\frac{\sin \left(N k_{0} \eta^{(0)} d\right)}{k_{0} \eta^{(0)} d} & \text { for } \eta^{(0)} \neq 0, \\
N & \text { for } \eta^{(0)}=0,\end{cases} \\
& P_{2}^{(0)}= \begin{cases}\cos \left(N k_{0} \eta^{(0)} d\right) & \text { for } \eta^{(0)} \neq 0, \\
1 & \text { for } \eta^{(0)}=0,\end{cases} \\
& P_{3}^{(0)}= \begin{cases}-i k_{0} d\left(\eta^{(0) 2}+\eta_{\text {in }} \eta_{\text {out }}\right) & \text { for } \eta^{(0)} \neq 0, \\
-i k_{0} d \eta_{\text {in }} \eta_{\text {out }} & \text { for } \eta^{(0)}=0,\end{cases}
\end{aligned}
$$

and

$$
\begin{aligned}
& \delta P_{1}= \begin{cases}\frac{p N \Delta K}{k_{0} \eta^{(0)}}+\mathrm{O}\left[(N \Delta K d)^{2}\right] & \text { for } \eta^{(0)} \neq 0, \\
\mathrm{O}\left[N^{3}\left(k_{0} d\right)^{6}\right] & \text { for } \eta^{(0)}=0,\end{cases} \\
& \delta P_{2}= \begin{cases}-\sin \left(N k_{0} \eta^{(0)} d\right) N \Delta K d & \text { for } \eta^{(0)} \neq 0, \\
+\mathrm{O}\left[(N \Delta K d)^{2}\right] & \text { for } \eta^{(0)}=0, \\
\mathrm{O}\left[N^{2}\left(k_{0} d\right)^{6}\right] & \end{cases} \\
& \delta P_{3}=\left(\eta_{\text {out }}-\eta_{\text {in }}\right) \frac{\sigma\left(k_{0} d\right)^{2}}{2}+\mathrm{O}\left[\left(k_{0} d\right)^{3}\right],
\end{aligned}
$$

where

$$
p=\left(\cos \left(N k_{0} \eta^{(0)} d\right)-\frac{\sin \left(N k_{0} \eta^{(0)} d\right)}{N k_{0} \eta^{(0)} d}\right) .
$$

The transmission difference for $\eta^{(0)} \neq 0$ is

$$
\Delta T=2 T_{0} \operatorname{Re}\left(t_{0} \tau_{1}\right) N \Delta K d+2 T_{0} \operatorname{Re}\left(t_{0}\right) \tau_{2} k_{0} d+\mathrm{O}\left[\left(k_{0} d\right)^{2}\right]
$$

and for $\eta^{(0)} \rightarrow 0$ is of the form

$$
\Delta T=2 T_{0} \operatorname{Re}\left(t_{0}\right) \tau_{3} N\left(k_{0} d\right)^{2}+\mathrm{O}\left[N\left(k_{0} d\right)^{3}\right],
$$

where

$$
\begin{aligned}
\tau_{1} & =\frac{\eta_{\text {in }}+\eta_{\text {out }}}{2 \eta_{\text {out }}} \sin \left(N k_{0} \eta^{(0)} d\right)+i p \frac{\eta^{(0) 2}+\eta_{\text {in }} \eta_{\text {out }}}{2 \eta_{\text {out }} \eta^{(0)}}, \\
\tau_{2} & =\frac{\eta_{\text {in }}-\eta_{\text {out }}}{4 \eta_{\text {out }} \eta^{(0)}} \sigma \sin \left(N k_{0} \eta^{(0)} d\right), \\
\tau_{3} & =\frac{\eta_{\text {in }}-\eta_{\text {out }}}{4 \eta_{\text {out }}} \sigma .
\end{aligned}
$$


[1] A. K. Sarychev and V. M. Shalaev, Electrodynamics of Metamaterials (World Scientific, Singapore, 2007).

[2] V. G. Veselago, Sov. Phys. Usp. 10, 509 (1968).

[3] D. R. Smith, W. J. Padilla, D. C. Vier, S. C. Nemat-Nasser, and S. Schultz, Phys. Rev. Lett. 84, 4184 (2000).

[4] Y. Liua and X. Zhang, Chem. Soc. Rev. 40, 2494 (2011).

[5] C. M. Soukoulis and M. Wegener, Nat. Photonics 5, 523 (2011).

[6] N. I. Zheludev and Y. S. Kivshar, Nat. Mater. 11, 917 (2011).

[7] M. Choi, S. H. Lee, Y. Kim, S. B. Kang, J. Shin, M. H. Kwak, K.-Y. Kang, Y.-H. Lee, N. Park, and B. Min, Nature (London) 470, 369 (2011).

[8] P.-Y. Chen, M. Farhat, and A. Alu, Phys. Rev. Lett. 106, 105503 (2011).

[9] H.-T. Chen, H. Yang, R. Singh, J. F. O’Hara, A. K. Azad, S. A. Trugman, Q. X. Jia, and A. J. Taylor, Phys. Rev. Lett. 105, 247402 (2010)

[10] A. Poddubny, I. V. Iorsh, P. A. Belov, and Y. S. Kivshar, Nat. Photonics 7, 948 (2013).

[11] J. C. Ginn, I. Brener, D. W. Peters, J. R. Wendt, J. O. Stevens, P. F. Hines, L. I. Basilio, L. K. Warne, J. F. Ihlefeld, P. G. Clem, and M. B. Sinclair, Phys. Rev. Lett. 108, 097402 (2012).

[12] S. H. Lee, M. Choi, T.-T. Kim, S. Lee, M. Liu, X. Yin, H. K. Choi, S. S. Lee, C.-G. Choi, S.-Y. Choi, X. Zhang, and B. Min, Nat. Mater. 11, 936 (2012).

[13] F. J. Rodriguez-Fortuno, A. Vakil, and N. Engheta, Phys. Rev. Lett. 112, 033902 (2014).

[14] M. Lapine, I. V. Shadrivov, D. A. Powell, and Y. S. Kivshar, Nat. Mater. 11, 30 (2012).

[15] C. Pfeiffer and A. Grbic, Phys. Rev. Lett. 110, 197401 (2013).

[16] S. Sun, Q. He, S. Xiao, Q. Xu, X. Li, and L. Zhou, Nat. Mater. 11, 426 (2012).

[17] E. Plum, X.-X. Liu, V. A. Fedotov, Y. Chen, D. P. Tsai, and N. I. Zheludev, Phys. Rev. Lett. 102, 113902 (2009).

[18] D. R. Smith, D. C. Vier, Th. Koschny, and C. M. Soukoulis, Phys. Rev. E 71, 036617 (2005).

[19] R. Zhao, Th. Koschny, and C. M. Soukoulis, Opt. Express 18, 14553 (2010).

[20] A. Andryieuski, R. Malureanu, and A. V. Lavrinenko, Phys. Rev. B 80, 193101 (2009).

[21] A. Andryieuski, C. Menzel, C. Rockstuhl, R. Malureanu, F. Lederer, and A. Lavrinenko, Phys. Rev. B 82, 235107 (2010).

[22] X.-X. Liu and A. Alu, Phys. Rev. B 87, 235136 (2013).

[23] C. R. Simovski, J. Opt. 13, 013001 (2011).

[24] A. Alu, Phys. Rev. B 84, 075153 (2011).

[25] J. Petschulat, C. Menzel, A. Chipouline, C. Rockstuhl, A. Tunnermann, F. Lederer, and T. Pertsch, Phys. Rev. A 78, 043811 (2008).

[26] J. Petschulat, A. Chipouline, A. Tunnermann, T. Pertsch, C. Menzel, C. Rockstuhl, T. Paul, and F. Lederer, Phys. Rev. B 82, 075102 (2010).

[27] A. V. Novitsky, V. M. Galynsky, and S. V. Zhukovsky, Phys. Rev. B 86, 075138 (2012).

[28] C. Fietz, J. Opt. Soc. Am. B 30, 1937 (2013).

[29] I. Tsukerman, J. Opt. Soc. Am. B 28, 577 (2011).

[30] A. Ciattoni and C. Rizza, Phys. Rev. B 91, 184207 (2015).
[31] M. A. Gorlach, T. A. Voytova, M. Lapine, Y. S. Kivshar, and P. A. Belov, Phys. Rev. B 93, 165125 (2016).

[32] G. Castaldi, V. Galdi, A. Alu, and N. Engheta, Phys. Rev. Lett. 108, 063902 (2012).

[33] M. Moccia, G. Castaldi, V. Galdi, A. Alu, and N. Engheta, Optica 3, 179 (2016).

[34] A. V. Chebykin, M. A. Gorlach, and P. A. Belov, Phys. Rev. B 92, 045127 (2015).

[35] H. Herzig Sheinfux, I. Kaminer, Y. Plotnik, G. Bartal, and M. Segev, Phys. Rev. Lett. 113, 243901 (2014).

[36] A. Andryieuski, A. V. Lavrinenko, and S. V. Zhukovsky, Nanotechnology 26, 184001 (2015).

[37] S. V. Zhukovsky, A. Andryieuski, O. Takayama, E. Shkondin, R. Malureanu, F. Jensen, and A. V. Lavrinenko, Phys. Rev. Lett. 115, 177402 (2015).

[38] D. R. Smith and D. Schurig, Phys. Rev. Lett. 90, 077405 (2003).

[39] Z. Jacob, L. V. Alekseyev, and E. Narimanov, Opt. Express 14, 8247 (2006).

[40] P. Shekhar, J. Atkinson, and Z. Jacob, Nano Convergence 1, 14 (2014).

[41] T. Repan, A. V. Lavrinenko, and S. V. Zhukovsky, Opt. Express 23, 25350 (2015).

[42] A. V. Chebykin, V. E. Babicheva, I. V. Iorsh, A. A. Orlov, P. A. Belov, and S. V. Zhukovsky, Phys. Rev. A 93, 033855 (2016).

[43] S. V. Zhukovsky, A. Andryieuski, J. E. Sipe, and A. V. Lavrinenko, Phys. Rev. B 90, 155429 (2014).

[44] A. A. Krokhin, J. Arriaga, L. N. Gumen, and V. P. Drachev, Phys. Rev. B 93, 075418 (2016).

[45] A. N. Borzdov, in Advances in Electromagnetics of Complex Media and Metamaterials, edited by S. Zouhdi, A. Sihvola, and M. Arsalane, NATO Science Series, Series II, Vol. 89 (Kluwer Academic, Dordrecht, 2003), p. 259.

[46] L. M. Barkovsky and A. N. Furs, Operator Methods to Describe Optical Fields in Complex Media (Belaruskaya Navuka, Minsk, 2003).

[47] L. M. Barkovskii, G. N. Borzdov, and A. V. Lavrinenko, J. Phys. A 20, 1095 (1987).

[48] L. M. Barkovskii, G. N. Borzdov, and F. I. Fedorov, J. Mod. Opt. 37, 85 (1990).

[49] G. N. Borzdov, J. Math. Phys. 38, 6328 (1997).

[50] G. N. Borzdov, Opt. Commun. 94, 159 (1992).

[51] G. N. Borzdov, in Advances in Complex Electromagnetic Materials, edited by A. Priou, A. Sihvola, S. Tretyakov, and A. Vinogradov (Kluwer Academic, Dordrecht, 1997), p. 71

[52] F. I. Fedorov, Theory of Gyrotropy (Nauka i Tekhnika, Minsk, 1976).

[53] F. I. Fedorov, Optics of Anisotropic Media (Akademia Nauk BSSR, Minsk, 1958).

[54] A. V. Lavrinenko and V. V. Zhilko, Microwave Opt. Technol. Lett. 15, 54 (1997).

[55] V. A. Markel and J. C. Schotland, J. Opt. 12, 015104 (2010).

[56] S. A. Tretyakov and A. A. Sochava, Prog. Electromagn. Res. 9, 157 (1994).

[57] C. F. Bohren and D. R. Huffman, Absorption and Scattering of Light by Small Particles (Wiley, New York, 1998). 